\title{
Indexing and Matching of Video Shots Based on Motion and Color Analysis
}

\author{
Ying Chen, Weiming $\mathrm{Hu}$, and Xianglin Zeng \\ National Laboratory of Pattern Recognition (NLPR) \\ Institute of Automation, Chinese Academy of Sciences \\ Beijing, People's Republic of China \\ Email: \{ychen,wmhu,xlzeng\}@nlpr.ia.ac.cn
}

\author{
Wanqing Li \\ SITACS, Faculty of Informatics \\ University of Wollongong, Australia \\ Email: wanqing@uow.edu.au
}

\begin{abstract}
This paper concerns two fundamental issues in video shots retrieval: key frame identification and similarity measurement between the key frames. We propose a simple key frame extraction algorithm based on optical flow. The algorithm emphasizes the motion extremum in the shot. Color histograms in HSV color space are adopted to describe the content of the extracted key frames and a new model is proposed to measure the similarity between the key frames from different shots. Preliminary experiments have shown that the proposed method outperforms existing ones in retrieving sport video shots.
\end{abstract}

Keywords-indexing, matching, key frame, optical flow

\section{INTRODUCTION}

The proliferation of multimedia content for both entertainment and professional services in the recent years has fueled the ever increasingly demand for efficient retrieval of the content. While technologies for text-based information retrieval is becoming mature and research in image retrieval is progressing at a fast pace, video retrieval still remains challenging due to the huge amount of data involved in any meaningful video indexing and matching. Much research is desired before we could have video sequences retrieved as efficiently as the nowaday retrieval of text information.

In video retrieval, video indexing and matching are two key technologies. Video sequences are generally segmented into a series of shots either manually or automatically by means of boundary identification [8], [14], [15], [16], each shot is composed of a group of correlative frames, referred as GOF. The shots are further summarized into a compact representation for indexing and matching.

There are two general approaches to summarizing a shot: color histogram based and key frame based. A commonly used color histogram based approach is the alpha-trimmed average color histogram which captures the statistical information of all frames in a GOF [3]. Its variants can be found in [4], [17]. In key-frame based approach, a natural idea is to consider the first, middle, and/or last frame as the shot's key frames [12] without exploring any motion information in the shot. In [9], [13], motion-based analysis has been employed for picking up more representative key frames. Especially, [13] computed the optical flow of each frame, and then, used an intuitionistic motion function to locate the key frames where the motion function has its minima.

Although the color histogram approach ignores the motion information contained in a shot, it is an effective statistical representation of the image content for matching. A mixture of the color histogram and key frame extraction would take the advantages of both approaches. The color histograms of the extracted key frames are expected to be more representative. The distance of the color histograms between query key frames and data key frames can be adopted to define the similarity between the query shot and data shots. As we know, the distance function usually has a quadratic form, $O\left(N^{2}\right)$ of complexity for two $N$-dimensional color histograms. Though this can be improved to $O(N)$ at best, some additional expenses are necessary, such as the pretreatment by diagonalizing the quadratic form. Hence, it is essential to reduce the computation when the similarity metric is applied to video retrieval.

Inspired by [13], we propose in this paper an improved algorithm to extract key frames of a GOF by analyzing its optical flows. The algorithm emphasizes the motion extremum in the shots. Color histogram in HSV color space is adopted to describe the content of the extracted key frames and a new geometric representation of similarity model has been present.

The rest of the paper is organized as follows. Section 2 gives a review of the optical flow method used in this paper. Section 3 describes the steps of key frame extraction. Section 4 discusses the similarity model in detail. Preliminary experimental results on the retrieval of sport video shots are shown and analyzed in Section 5. Section 6 concludes the paper.

\section{REVIEW OF OPTICAL FLOW}

An optical flow is the distribution of apparent motion of brightness patterns between two images. Many methods have been proposed in the past [1]. In this paper, we adopt the wellknown Lucas-Kanade's algorithm which is based on spatial and temporal gradients.

\section{A. The Gradient Constraint Equation}

Let the function $f=I(x, y, t)$ be the image brightness at point $(x, y)$ in the image plane at time $t$. Assume that $I(x, y, t)$ 
is differentiable and the change of brightness over time for each point is constant, i.e.

$$
\frac{d I}{d t}=0 .
$$

Notice that $x$ and $y$ also can be regarded as the function of $t$ respectively, by the chain rule for differentiation we have the gradient constraint equation [5]:

$$
(\nabla I)^{T} \cdot \vec{v}+I_{t}=0 .
$$

Where

$$
\begin{gathered}
I_{t}=\frac{\partial I}{\partial t}, \\
(\nabla I)^{T}=\left(\frac{\partial I}{\partial x}, \frac{\partial I}{\partial y}\right), \\
\vec{v}=(\mu, \nu)^{T}=\left(\frac{d x}{d t}, \frac{d y}{d t}\right)^{T} .
\end{gathered}
$$

Equation (2) has two variables about the optical flow $\vec{v}=$ $(\mu, \nu)$. To solve it, more constraints about the two variables are required.

\section{B. Lucas And Kanade's Algorithm}

Lucas and Kanade's algorithm [10], [11] is based on a weighted local least square. It assumes that the $(\mu, \nu)$ is constant for an small enough region $\mathcal{D}$ and minimizes

$$
\sum_{p} \mathcal{W}^{2}(p)\left((\nabla I)^{T} \cdot \vec{v}+I_{t}\right)^{2},
$$

where $p \in \mathcal{D}$ is the pixel point and $\mathcal{W}(x)$ is the weighted function. For $n$ points $p_{i} \in \mathcal{D}$ at time $t$, let

$$
\begin{aligned}
& \mathcal{I}=\left(\nabla I\left(p_{1}\right), \cdots, \nabla I\left(p_{n}\right)\right)^{T}, \\
& \mathcal{I}_{t}=-\left(I_{t}\left(p_{1}\right), \cdots, I_{t}\left(p_{n}\right)\right)^{T},
\end{aligned}
$$

and

$$
\mathcal{W}=\left(\begin{array}{ccccc}
\mathcal{W}\left(p_{1}\right) & 0 & \cdots & 0 & 0 \\
0 & \ddots & \ddots & \ddots & 0 \\
\vdots & \ddots & \mathcal{W}\left(p_{i}\right) & \ddots & \vdots \\
0 & \ddots & \ddots & \ddots & 0 \\
0 & 0 & \cdots & 0 & W\left(p_{n}\right)
\end{array}\right)
$$

By the derivative of (6) with respect to $\mu$ and $\nu$, the $\vec{v}=$ $(\mu, \nu)^{T}$ is given by

$$
\mathcal{I}^{T} \mathcal{W}^{2} \mathcal{I} \vec{v}=\mathcal{I}^{T} \mathcal{W}^{2} \mathcal{I}_{t}
$$

Obviously, $\mathcal{I}^{T} \mathcal{W}^{2} \mathcal{I}$ is a $2 \times 2$ matrix and equals to

$$
\left(\begin{array}{cc}
\sum \mathcal{W}^{2}\left(p_{i}\right) I_{x}^{2}\left(p_{i}\right) & \sum \mathcal{W}^{2}\left(p_{i}\right) I_{x}\left(p_{i}\right) I_{y}\left(p_{i}\right) \\
\sum \mathcal{W}^{2}\left(p_{i}\right) I_{y}\left(p_{i}\right) I_{x}\left(p_{i}\right) & \sum \mathcal{W}^{2}\left(p_{i}\right) I_{y}^{2}\left(p_{i}\right)
\end{array}\right) .
$$

If $\left|\mathcal{I}^{T} \mathcal{W}^{2} \mathcal{I}\right| \neq 0$, then

$$
\vec{v}=\left(\mathcal{I}^{T} \mathcal{W}^{2} \mathcal{I}\right)^{-1} \mathcal{I}^{T} \mathcal{W}^{2} \mathcal{I}_{t}
$$

\section{EXTRACTION OF KEY FRAMES}

The proposed method to extract key frames from a GOF consists of three main steps.

\section{A. Compute Optical Flow}

Assume that the given GOF has $N+1$ frames. $N$ optical flow fields $\left\{\mathcal{G}_{1}, \cdots, \mathcal{G}_{N}\right\}$ will be computed by Lucas-Kanade's algorithm.

\section{B. Define Motion Function}

Define the motion function $M(i)$ for $\mathcal{G}_{i}$ as,

$$
M(i)=\frac{\sum_{(x, y) \in \mathcal{G}_{i}} \sqrt{\mu^{2}(x, y)+\nu^{2}(x, y)}}{P \max _{(x, y) \in \mathcal{G}_{i}} \sqrt{\mu^{2}(x, y)+\nu^{2}(x, y)}},
$$

where $i=1, \cdots, N$ and $P$ is the total number of pixel points in $\mathcal{G}_{i}$.

\section{Select Key Frames}

Scan the curve $M(i)$ from 1 to $N$ about $i$, then key frames are found at places where the motion function $M(i)$ has its global maximum and minimum.

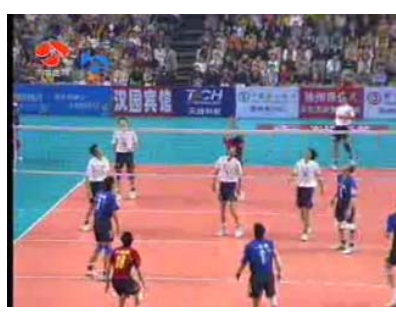

(a) Frame 2654

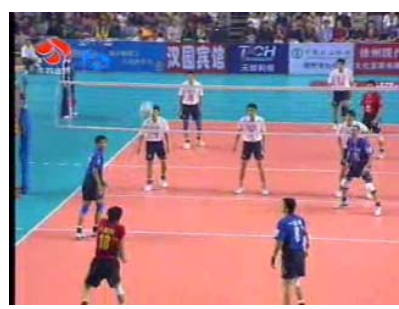

(c) Frame 2722

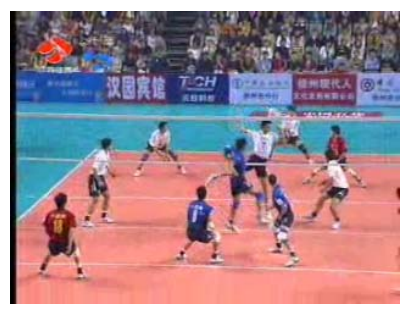

(e) Frame 2790

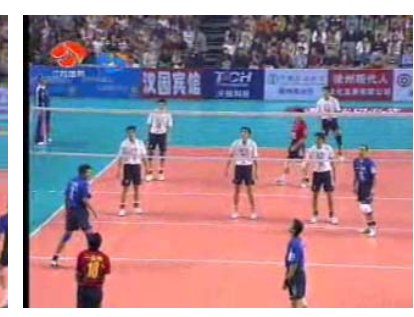

(b) Frame 2688

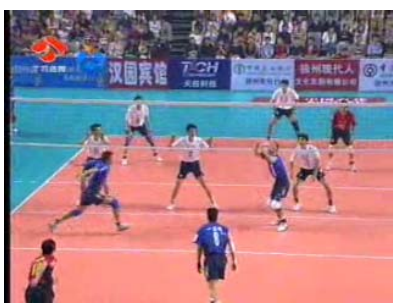

(d) Frame 2756

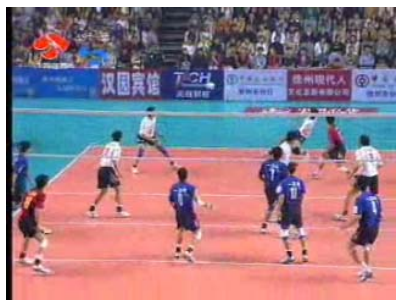

(f) Frame 2824
Fig. 1. The sample frames of an example GOF. 


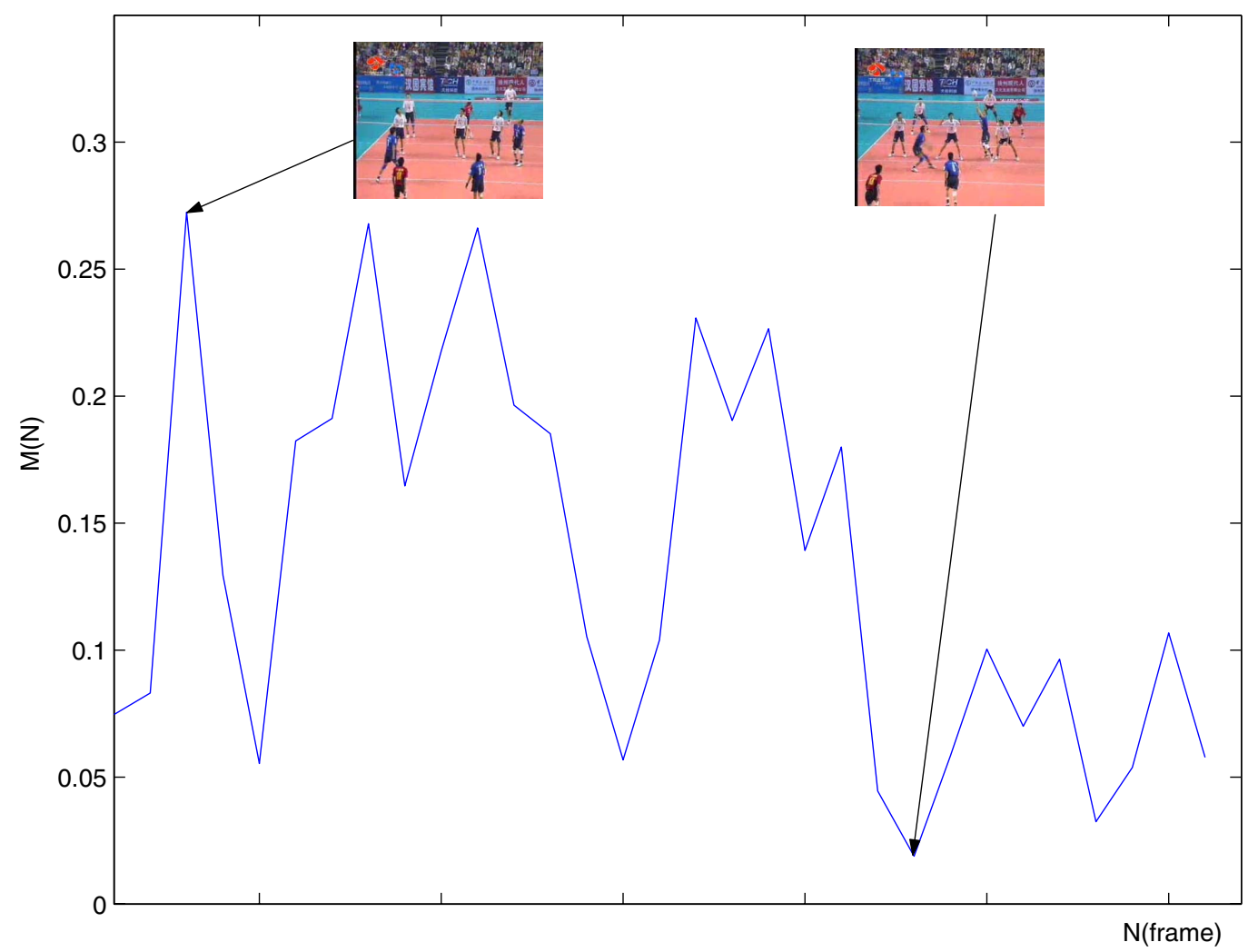

Fig. 2. Motion function, $M(\cdot)$ and the extracted key frames of the example GOF.

\section{An Example}

Fig. 1 illustrates an example GOF taken from a volleyball video sequence. The corresponding motion function, $M(\cdot)$, and the extracted key frames are shown in Fig. 2.

\section{Similarity Measurement}

In this section, we present a new model to measure the similarity between the key frames extracted from a query shot and data shots by using color histogram of the key frames in hue-saturation-brightness (HSV) color space since HSV color space often outperforms RGB color space in many applications [7] due to its improved perceptual uniformity.

\section{A. Quantize HSV Cone Space}

Video sequences are often stored in the standard red-greenblue (RGB) color space. Let $(r(x, y), g(x, y), b(x, y))$ be a triple to represent the color in RGB color space for the pixel point at $(x, y)$. We convert RGB color space into HSV color space through the following equations [6]:

$$
\begin{gathered}
v=\max (r, g, b), \\
s=\frac{v-\min (r, g, b)}{v},
\end{gathered}
$$

$$
h=\left\{\begin{array}{l}
5+b^{\prime} \text { if } r=\max (r, g, b) \text { and } g=\min (r, g, b) \\
1-g^{\prime} \text { if } r=\max (r, g, b) \text { and } g \neq \min (r, g, b) \\
1+r^{\prime} \text { if } g=\max (r, g, b) \text { and } b=\min (r, g, b) \\
3-b^{\prime} \text { if } g=\max (r, g, b) \text { and } b \neq \min (r, g, b) \\
3+g^{\prime} \text { if } b=\max (r, g, b) \text { and } r=\min (r, g, b) \\
5-r^{\prime} \text { otherwise, }
\end{array}\right.
$$

where

$$
\begin{aligned}
r^{\prime} & =\frac{v-r}{v-\min (r, g, b)}, \\
g^{\prime} & =\frac{v-g}{v-\min (r, g, b)},
\end{aligned}
$$

and

$$
b^{\prime}=\frac{v-b}{v-\min (r, g, b)} .
$$

The $(h, s, v)$ is the color triple in HSV color space. Notice that the HSV color space is always treated as a cone [2]: the Cartesian coordinates $(s v \cos h, s v \sin h, v)$ mapped from $(h, s, v)$ can be used to measure color in human vision. In this paper, a 3D Cartesian coordinate system with 20 values for $X$ (the hue), 3 values for $\mathrm{Y}$ (the saturation), and 3 values for $\mathrm{Z}$ (the brightness) is used to quantize the HSV cone space. 


\section{B. Construct Color Histogram}

For $\mathcal{B}=20 \times 3 \times 3=180$ color bins, assume that the key frame's size is $W \times H$ and $c(x, y)$ denotes the color triple $(h, s, v)$ at $(x, y)$, then the color histogram of the key frame is calculated:

$$
p_{b}=\frac{1}{T} \sum_{x=0}^{W-1} \sum_{y=0}^{H-1} \delta(c(x, y), b),
$$

where

$$
T=\sum_{b=1}^{\mathcal{B}} \sum_{x=0}^{W-1} \sum_{y=0}^{H-1} \delta(c(x, y), b),
$$

and

$$
\delta(c(x, y), b)= \begin{cases}1, & \text { if } c(x, y) \in \text { the } b \text {-th bin } \\ 0, & \text { otherwise }\end{cases}
$$

The $\mathcal{B}$-dimensional vector

$$
\left(p_{1}, \cdots, p_{\mathcal{B}}\right), \sum_{i=1}^{\mathcal{B}} p_{i}=1
$$

describes the color distribution of the key frame.

\section{Build Similarity Model}

The purpose of the similarity model is to measure how similar of two color histograms, $\mathcal{F}$ and $\mathcal{K}$, which can be decomposed into the similarity between bins in the same histogram and bins from two different histograms.

1) Distance Between Bins in One Histogram: We consider the entire database as the training set in which the principal color value of the $i$-th bin is represented by the point $m_{i}=$ $\left(s_{i} v_{i} \cos h_{i}, s_{i} v_{i} \sin h_{i}, v_{i}\right)$ whose frequency of occurrence is maximum in this bin, where $i=1, \cdots, \mathcal{B}$. The distance between the $i$-th bin and the $j$-th bin is defined as the distance between $m_{i}$ and $m_{j}$ :

$$
\begin{aligned}
d_{i, j}^{\prime}= & \left\|m_{i}-m_{j}\right\| \\
= & {\left[\left(s_{i} v_{i} \cos h_{i}-s_{j} v_{j} \cos h_{j}\right)^{2}\right.} \\
& +\left(s_{i} v_{i} \sin h_{i}-s_{j} v_{j} \sin h_{j}\right)^{2} \\
& \left.+\left(v_{i}-v_{j}\right)^{2}\right]^{\frac{1}{2}}
\end{aligned}
$$

Let $d_{\max }^{\prime}=\max _{(i, j)}\left\{d_{i j}^{\prime}\right\}$, then $d_{i j}^{\prime}$ is normalized as:

$$
d_{i j}=\frac{d_{i, j}^{\prime}}{d_{\max }^{\prime}}
$$

2) Distance Between Bins in Two Histograms: Let

$$
\mathcal{F}=\left(f_{1}, \cdots, f_{\mathcal{B}}\right), \sum_{i=1}^{\mathcal{B}} f_{i}=1,
$$

and

$$
\mathcal{K}=\left(k_{1}, \cdots, k_{\mathcal{B}}\right), \sum_{i=1}^{\mathcal{B}} k_{i}=1 .
$$

Without loss of generality, we consider the following two cases for any given $i$ and $j$, where $i \neq j \in\{1, \cdots, \mathcal{B}\}$.

- Case 1: As shown in Fig. 3, the area of the trapezoid $f_{i} f_{j} k_{i} k_{j}$ denotes the distance of the four bins of the two histograms:

$$
\begin{aligned}
\mathcal{D}_{i j} & =S_{f_{i} f_{j} k_{i} k_{j}} \\
& =\frac{1}{2}\left(k_{i}+k_{j}-f_{i}-f_{j}\right) d_{i j} .
\end{aligned}
$$

It is obvious that the trapezoid become a degenerate triangle when $f_{i}=k_{i}$ or $f_{j}=k_{j}$.

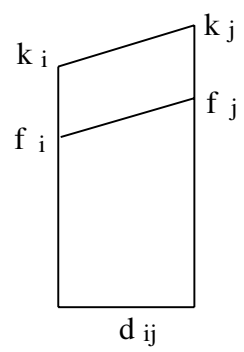

Fig. 3. Case 1: Line segments $f_{i} f_{j}$ and $k_{i} k_{j}$ don't intersect at an inner point.

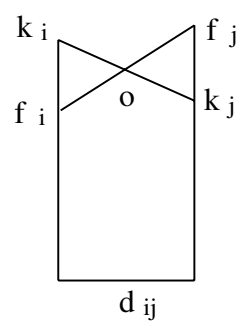

Fig. 4. Case 2: Line segments $f_{i} f_{j}$ and $k_{i} k_{j}$ intersect at an inner point.

- Case 2: As shown in Fig. 4, let the intersect point be $o$. In this case, we determine the distance as follows:

$$
\begin{aligned}
\mathcal{D}_{i j} & =S_{o f_{i} k_{i}}+S_{o f_{j} k_{j}} \\
& =\frac{1}{2}\left(k_{i}-f_{i}\right) o_{\perp k_{i} f_{i}}+\frac{1}{2}\left(f_{j}-k_{j}\right) o_{\perp f_{j} k_{j}},
\end{aligned}
$$

where $o_{\perp k_{i} f_{i}}$ denotes the vertical line segment from point $o$ to $k_{i} f_{i}$ and $o_{\perp f_{j} k_{j}}$ denotes the vertical line 
segment from point $o$ to $f_{j} k_{j}$. According to the geometric relationship, we have

$$
o_{\perp k_{i} f_{i}}=\frac{k_{i}-f_{i}}{f_{j}-f_{i}+k_{i}-k_{j}} d_{i j}
$$

and

$$
o_{\perp f_{j} k_{k}}=\frac{f_{j}-k_{j}}{f_{j}-f_{i}+k_{i}-k_{j}} d_{i j} .
$$

So that

$$
\mathcal{D}_{i j}=\frac{d_{i j}}{2\left(f_{j}-f_{i}+k_{i}-k_{j}\right)}\left[\left(k_{i}-f_{i}\right)^{2}+\left(f_{j}-k_{j}\right)^{2}\right] .
$$

3) Total Distance Between Two Histograms: Assume that the $i$-th bin corresponds to the interval $(X, Y, Z)$ in the HSV cone space. The distance of the $i$-th bin between two histograms, $\widetilde{\mathcal{D}}_{i}$, is defined as the average of its conjoint bins.

$$
\begin{aligned}
\widetilde{\mathcal{D}_{i}=} & \frac{1}{6}\left(\mathcal{D}_{(X, Y, Z)(X-1, Y, Z)}+\mathcal{D}_{(X, Y, Z)(X+1, Y, Z)}\right. \\
& +\mathcal{D}_{(X, Y, Z)(X, Y-1, Z)}+\mathcal{D}_{(X, Y, Z)(X, Y+1, Z)} \\
& +\mathcal{D}_{(X, Y, Z)(X, Y, Z-1)}+\mathcal{D}_{(X, Y, Z)(X, Y, Z+1)} .
\end{aligned}
$$

Fig. 5 illustrates the derivation of $\widetilde{\mathcal{D}_{i}}$.

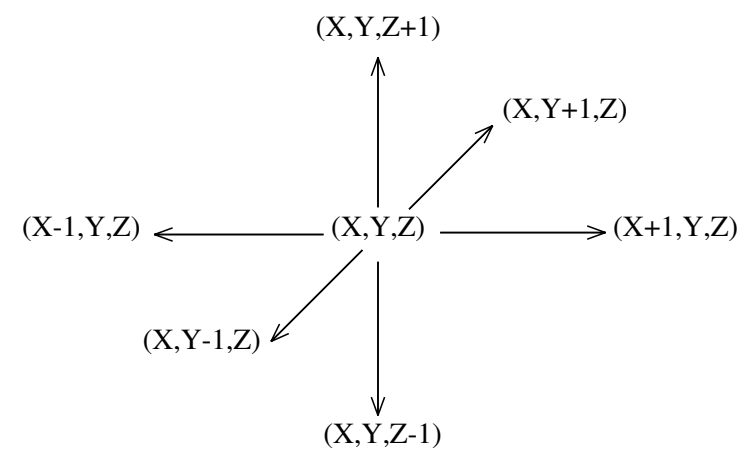

Fig. 5. The distance of the $i$-th bin between two histograms is the average of its conjoint bins. Here the index $X$ corresponds to the $s v$ cosh direction in HSV cone space, the index $Y$ to the $s v \sinh$ direction, while the index $Z$ to the $v$ direction.

Now, the total distance between two color histograms $\mathcal{F}$ and $\mathcal{K}$ is given as:

$$
\mathcal{D}(\mathcal{F}, \mathcal{K})=\sum_{i=1}^{\mathcal{B}} \widetilde{D_{i}}
$$

Obviously, the best match result for the query is the one with the smallest total distance.

\section{EXPERIMENTS AND DISCUSSION}

\section{A. Performance indicators}

To evaluate the performance of the proposed method for the retrieval of video shots, the average normalized modified retrieval rank (ANMRR) and the average recall (AR), which were developed in [18], are chosen as the benchmark indicators. The value of ANMRR determines the rank of the correct shots unretrieved and the value of AR determines the rate of the correct shots retrieved. The lower the value of the ANMRR, the better the performance. In contrast, the higher the value of the AR, the better the performance.

Let $\left\{q_{1}, \cdots, q_{S}\right\}$ denote the set of the query shots. For any $q_{i}$, the set $G\left(q_{i}\right)$ denotes the ground truth shots and

$$
K_{q_{i}}=\min \left\{4 \times\left\|G\left(q_{i}\right)\right\|, 2 \times \max _{i}\left\|G\left(q_{i}\right)\right\|\right\}
$$

denotes the value of the threshold, where $\|\sharp\|$ is the number of the elements in the set $\sharp$. Then the set $R\left(q_{i}\right)$ denotes the items correctly retrieved in the top $K_{q_{i}}$ results. So the value of the AR and the ANMRR are calculated as:

$$
A R=\frac{1}{S} \sum_{i=1}^{S} \frac{\left\|R\left(q_{i}\right)\right\|}{\left\|G\left(q_{i}\right)\right\|},
$$

and

$$
A N M R R=\frac{1}{S} \sum_{i=1}^{S} \frac{\sum_{m=1}^{\left\|G\left(q_{i}\right)\right\|} \frac{\operatorname{Rank}\left(g_{m}\right)}{\left\|G\left(q_{i}\right)\right\|}-\frac{\left\|G\left(q_{i}\right)\right\|}{2}-\frac{1}{2}}{K_{q_{i}}-\frac{\left\|G\left(q_{i}\right)\right\|}{2}+\frac{1}{2}},
$$

where for $g_{m} \in G\left(q_{i}\right)$, the $\operatorname{Rank}\left(g_{m}\right)$ equals to the order in the top $K_{q_{i}}$ retrievals if $g_{m} \in R\left(q_{i}\right)$, while $K_{q_{i}}+1$ if $g_{m} \notin R\left(q_{i}\right)$.

\section{B. Experimental results}

98 pre-cut sport video shots were used for test. These shots were extracted from the video sequences of volleyball, basketball and tennis matching. Table I shows the ANMRR and AR values for the proposed method and the method with the extraction of key frames described in [13] and the quadratic form metric. It can be seen that our method achieved better performance.

TABLE I

ANMRR VALUES AND AR VALUES OF THE PROPOSED METHOD AND METHOD SYNTHESIZING [13] AND CONVENTIONAL QUADRATIC FORM METRIC

\begin{tabular}{|c|c|c|}
\hline & ANMRR & AR \\
\hline Volleyball Video & & \\
\hline Old method & 0.5079 & 0.5560 \\
\hline Proposed method & 0.5317 & 0.6667 \\
\hline Basketball Video & & \\
\hline Old method & 0.2464 & 0.9000 \\
\hline Proposed method & 0.2390 & 0.9000 \\
\hline Tennis Video & & \\
\hline Old method & 0.4500 & 0.7000 \\
\hline Proposed method & 0.3375 & 0.9000 \\
\hline
\end{tabular}




\section{Discussion}

[13] defined the following motion function for the extraction of key frames:

$$
M(i)=\sum_{(x, y) \in \mathcal{G}_{i}}|\mu(x, y)|+|\nu(x, y)| .
$$

Compare equation (38) with equation (13), we can see that the latter has a geometric description about the magnitude of the optical flow field and considers a well-balanced relative relation between pixel points. In addition, because the optical flow only approximates the actual motion, we believe that not only the minimum but also the maximum of the motion function carries important information about the shots, whereas [13] ignored the maximum. In addition, the computational complexity of the proposed similarity matching is in the order of $O(N)$.

\section{CONCLUSION AND FUTURE WORK}

We have proposed an algorithm based on motion and color information for video shot retrieval. Generally speaking, A huge database is more convictive when the ANMRR and the $\mathrm{AR}$ are adopted to evaluate the performance of the retrieval. For this reason, our future work will focus on testing the proposed algorithm using a large video database and further improving its performance.

\section{ACKNOWLEDGMENT}

The authors would like to appreciate many other workmates for their valuable suggestion. This work is partly supported by NSFC (Grant No. 60373046 and 60520120099), Natural Science Foundation of Beijing (Grant No. 4041004).

\section{REFERENCES}

[1] J. L. Barron, D. J. Fleet, S. S. Beauchemin, and T. A. Burkitt, Performance of optical flow techniques. IEEE Computer Society Conference on Computer Vision and Pattern Recognition, pp. 236-242, 1992.

[2] S. Belongie, Ch. Carson, H. Greenspan and J. Malik, Recoginition of images in large databases using a learning framework. Technical Report 97-939, UC Berkeley CS Division, 1997.

[3] A. M. Ferman, S. Krishnamachari, A. M. Tekalp, M. A. Mottaleb, and R. Mehrotra, Group-of-frames/pictures color histogram descriptors for multimedia applications. IEEE Int. Conf. on Image Processing, pp. 6568, Sep. 2000.

[4] B. Gunsel and A. M. Tekalp, Content-based video abstraction. IEEE Int. Conf. Image Processing, Chicago, IL, pp. 237-240, 1998.

[5] B. K. P. Horn and B. Schunck, Determining Optical Flow. Artificial Intelligence, pp. 185-203, 1981.

[6] R. W. G. Hunt, Measuring Color. John Wiley and Sons, 1989.

[7] A. Jain, Fundamentals of digital image processing. Prentice Hall, 1989.

[8] M. S. Lee, Y. M. Yang, and S. W. Lee, Automatic video parsing using shot boundary detection and camera operation analysis. Pattern Recognit., vol. 34, no. 3, pp. 711-719, Mar. 2001.

[9] T. M. Liu, H. J. Zhang, and F. H. Q, A novel video key-frame-extraction algorithm based on perceived motion energy model. IEEE Trans. on Circuits and Systems for Video Techn., vol. 13, no. 10, Oct. 2003.

[10] B. Lucas and T. Kanade, An iterative image registration technique with an application to stereo vision. Seventh International Joint Conference on Artificial Intelligence, Vancouver, Canada, pp. 674C679, 1981.

[11] B. Lucas, Generalized image matching by the method of differences. $\mathrm{PhD}$ thesis, School of Computer Science, CarnegieC Mellon University, Pittsburgh, PA, 1984.

[12] A. Nagasaka and Y. Tanaka, Automatic video indexing and full-video search for object appearances. in Visual Database Systems II, 1992.
[13] W. Wolf, Key frame selection by motion analysis. IEEE Int. Conf. Acoust., Speech, and Signal Proc., 1996.

[14] W. Xiong, J. C. M. Lee, and R. H. Ma, Automatic video data structuring through shot partitioning and key frame selection. Machine Vision Application, vol. 10, no. 2, pp. 51-65, 1997.

[15] J. Yu and M. D. Srinath, An efficient method for scene cut detection. Pattern Recogn. Lett., vol. 22, no. 13, pp. 1379-1391, Nov. 2001.

[16] H. J. Zhang, A. Kankamhalli, and S. Smoliar, Automatic partitioning of full-motion video. ACM Multimedia System, New York: ACM Press, vol. 1, pp. 10-28, 1993.

[17] H. J. Zhang, J. Wu, D. Zhong, and S. W. Smoliar, An integrated system for content-based video retrieval and browsing. Pattern Recognit., vol. 30, no. 4, pp. 643-658, 1997.

[18] MPEG-7 visual part of experimentation Model (XM) version 2.0. MPEG-7 Output Document ISO/MPEG, Dec., 1999. 